\title{
Positive Solutions for Fourth-Order Nonlinear Differential Equation with Integral Boundary Conditions
}

\author{
Qi Wang, Yanping Guo, and Yude Ji \\ Hebei University of Science and Technology, Shijiazhuang, Hebei 050018, China \\ Correspondence should be addressed to Yanping Guo; guoyanping65@sohu.com
}

Received 5 November 2012; Accepted 8 January 2013

Academic Editor: Cengiz Çinar

Copyright (C) 2013 Qi Wang et al. This is an open access article distributed under the Creative Commons Attribution License, which permits unrestricted use, distribution, and reproduction in any medium, provided the original work is properly cited.

This paper investigates the existence and nonexistence of positive solutions for a class of fourth-order nonlinear differential equation with integral boundary conditions. The associated Green's function for the fourth-order boundary value problems is first given, and the arguments are based on Krasnoselskii's fixed point theorem for operators on a cone.

\section{Introduction}

In this paper, we consider the following fourth-order boundary value problems (BVPs) with integral boundary conditions

$$
\begin{aligned}
x^{(4)}(t)=w(t) f\left(t, x(t), x^{\prime \prime}(t)\right), \quad t \in(0,1), & \\
x(0) & =\int_{0}^{1} h_{1}(s) x(s) \mathrm{d} s, \\
x(1) & =\int_{0}^{1} k_{1}(s) x(s) \mathrm{d} s, \\
x^{\prime \prime}(0) & =\int_{0}^{1} h_{2}(s) x^{\prime \prime}(s) \mathrm{d} s, \\
x^{\prime \prime}(1) & =\int_{0}^{1} k_{2}(s) x^{\prime \prime}(s) \mathrm{d} s,
\end{aligned}
$$

where $w$ may be singular at $t=0$ and(or) $t=1, f$ : $[0,1] \times[0,+\infty) \times(-\infty, 0] \rightarrow[0,+\infty)$ is continuous, and $h_{1}, h_{2}, k_{1}, k_{2} \in L^{1}[0,1]$ are nonnegative.

The existence of solutions for nonlinear higher-order nonlocal BVPs has been studied by several authors, for example, see [1-9] and the references therein. However, there are a few papers dealing with the existence of positive solutions for the fourth-order boundary value problems with integral boundary conditions, see [7-15]. For the case of $h_{1}, h_{2}, k_{1}, k_{2}=0$, BVP (1) reduces to the following two-point BVP

$$
\begin{gathered}
x^{(4)}(t)=w(t) f\left(t, x(t), x^{\prime \prime}(t)\right), \quad t \in(0,1), \\
x(0)=x(1)=x^{\prime \prime}(0)=x^{\prime \prime}(1)=0 .
\end{gathered}
$$

A great deal of research has been devoted to the existence of solutions for problem (2) by using the Leray-Schauder continuation method, topological degree, and the method of lower and upper solutions.

For the case of $h_{1}=g, h_{2}=h, k_{1}=0, k_{2}=0$ or $h_{1}=$ $0, h_{2}=0, k_{1}=g$, and $k_{2}=h, \mathrm{BVP}(1)$ reduces to the following BVP with integral boundary conditions

$$
\begin{aligned}
& x^{(4)}(t)=w(t) f\left(t, x(t), x^{\prime \prime}(t)\right), \quad t \in(0,1), \\
& x(0)=\int_{0}^{1} g(s) x(s) \mathrm{d} s, \quad x(1)=0, \\
& x^{\prime \prime}(0)=\int_{0}^{1} h(s) x^{\prime \prime}(s) \mathrm{d} s, \quad x^{\prime \prime}(1)=0,
\end{aligned}
$$

or

$$
\begin{gathered}
x^{(4)}(t)=w(t) f\left(t, x(t), x^{\prime \prime}(t)\right), \quad t \in(0,1), \\
x(0)=0, \quad x(1)=\int_{0}^{1} g(s) x(s) \mathrm{d} s, \\
x^{\prime \prime}(0)=0, \quad x^{\prime \prime}(1)=\int_{0}^{1} h(s) x^{\prime \prime}(s) \mathrm{d} s .
\end{gathered}
$$


By using the fixed point theorem of cone expansion and compression of norm type, Zhang and Ge [11] have obtained the existence and multiplicity of positive solutions for BVP (3) and (4).

The following assumptions will stand throughout this paper:

(A1) $\omega$ is nonnegative, and $\omega \in L^{1}[0,1]$ may have singularities at $t=0$ and(or) $t=1$;

(A2) $f \in C([0,1] \times[0,+\infty) \times(-\infty, 0],[0,+\infty))$;

(A3) $h_{1}, h_{2}, k_{1}, k_{2} \in L^{1}[0,1]$ are nonnegative and

$$
\begin{gathered}
\mu_{1}=1-\int_{0}^{1} h_{1}(s) \mathrm{d} s>0, \\
\nu_{1}=1-\int_{0}^{1} k_{1}(s) \mathrm{d} s>0, \\
\mu_{2}=1-\int_{0}^{1} h_{2}(s) \mathrm{d} s>0, \\
\nu_{2}=1-\int_{0}^{1} k_{2}(s) \mathrm{d} s>0 .
\end{gathered}
$$

The paper is organized as follows. In Section 2, we provide some necessary background material such as the Krasnoselskii's fixed point theorem in cones. The associated Green's function for the fourth-order boundary value problem is first given, and we also look at some properties of the Green's function associated with the problem (1). In Section 3, the main results of problem (1) will be stated and proved. In Section 4, we give an example to illustrate how the main results can be used in practice.

\section{Preliminary Results}

In our main results, we will make use of the following lemmas.

Lemma 1 (in [16]). Let $\Omega_{1}$ and $\Omega_{2}$ be two bounded open sets in a real Banach space $E$, such that $\theta \in \Omega_{1}$ and $\overline{\Omega_{1}} \subset \Omega_{2}$. Let operator $A: P \cap\left(\overline{\Omega_{2}} \backslash \Omega_{1}\right) \rightarrow P$ be continuous, where $\theta$ denotes the zero element of $E$ and $P$ is a cone in $E$. Suppose that one of the two conditions

(1) $\|A x\| \leq\|x\|, \forall x \in P \cap \partial \Omega_{1}$ and $\|A x\| \geq\|x\|, \forall x \in$ $P \cap \partial \Omega_{2}$ or

(2) $\|A x\| \geq\|x\|, \forall x \in P \cap \partial \Omega_{1}$ and $\|A x\| \leq\|x\|, \forall x \in$ $P \cap \partial \Omega_{2}$ is satisfied. Then, $A$ has at least one fixed point in $P \cap\left(\overline{\Omega_{2}} \backslash \Omega_{1}\right)$.

Lemma 2. If $h, k \in L^{1}[0,1]$ are nonnegative, and $\mu=1-$ $\int_{0}^{1} h(s) d s>0, v=1-\int_{0}^{1} k(s) d s>0$, then for any $y \in C(0,1)$, the $B V P$

$$
\begin{gathered}
-x^{\prime \prime}(t)=y(t), \quad t \in(0,1), \\
x(0)=\int_{0}^{1} h(s) x(s) d s, \\
x(1)=\int_{0}^{1} k(s) x(s) d s,
\end{gathered}
$$

has a unique solution $x$ which is given by

$$
x(t)=\int_{0}^{1} \widetilde{H}(t, s) y(s) d s
$$

where

$$
\begin{gathered}
\widetilde{H}(t, s)=G(t, s)+\frac{m+\mu t}{m v+n \mu} \int_{0}^{1} k(\tau) G(s, \tau) d \tau \\
+\frac{n-v t}{m v+n \mu} \int_{0}^{1} h(\tau) G(s, \tau) d \tau, \\
G(t, s)= \begin{cases}s(1-t), & 0 \leq s \leq t \leq 1, \\
t(1-s), & 0 \leq t \leq s \leq 1,\end{cases} \\
m=\int_{0}^{1} s h(s) d s, \quad n=1-\int_{0}^{1} s k(s) d s .
\end{gathered}
$$

Proof. The general solution of $-x^{\prime \prime}(t)=y(t)$ can be written as

$$
x(t)=-\int_{0}^{t}(t-s) y(s) \mathrm{d} s+A t+B .
$$

Now, we solve for $A, B$ by $x(0)=\int_{0}^{1} h(s) x(s) \mathrm{d} s$ and $x(1)=$ $\int_{0}^{1} k(s) x(s) \mathrm{d} s$, it follows that

$$
\begin{aligned}
& B=-\int_{0}^{1} h(\tau) \int_{0}^{\tau}(\tau-s) y(s) \mathrm{d} s \mathrm{~d} \tau \\
& +A \int_{0}^{1} \tau h(\tau) \mathrm{d} \tau+B \int_{0}^{1} h(\tau) \mathrm{d} \tau \\
& -\int_{0}^{1}(1-s) y(s) \mathrm{d} s+A+B \\
& =-\int_{0}^{1} k(\tau) \int_{0}^{\tau}(\tau-s) y(s) \mathrm{d} s \mathrm{~d} \tau \\
& \quad+A \int_{0}^{1} \tau k(\tau) \mathrm{d} \tau+B \int_{0}^{1} k(\tau) \mathrm{d} \tau
\end{aligned}
$$

that is,

$$
\begin{aligned}
& A \int_{0}^{1} \tau h(\tau) \mathrm{d} \tau-B\left(1-\int_{0}^{1} h(\tau) \mathrm{d} \tau\right) \\
& \quad=\int_{0}^{1} h(\tau) \int_{0}^{\tau}(\tau-s) y(s) \mathrm{d} s \mathrm{~d} \tau \\
& A\left(1-\int_{0}^{1} \tau k(\tau) \mathrm{d} \tau\right)+B\left(1-\int_{0}^{1} k(\tau) \mathrm{d} \tau\right) \\
& \quad=\int_{0}^{1}(1-s) y(s) \mathrm{d} s-\int_{0}^{1} k(\tau) \int_{0}^{\tau}(\tau-s) y(s) \mathrm{d} s \mathrm{~d} \tau
\end{aligned}
$$


Solving the above equations, we get

$$
\begin{aligned}
& A=\frac{1}{m \nu+n \mu}\left[v \int_{0}^{1} h(\tau) \int_{0}^{\tau}(\tau-s) y(s) \mathrm{d} s \mathrm{~d} \tau\right. \\
& +\mu\left(\int_{0}^{1}(1-s) y(s) \mathrm{d} s\right. \\
& \left.\left.-\int_{0}^{1} k(\tau) \int_{0}^{\tau}(\tau-s) y(s) \mathrm{d} s \mathrm{~d} \tau\right)\right], \\
& B=\frac{1}{m v+n \mu}\left[m \left(\int_{0}^{1}(1-s) y(s) \mathrm{d} s\right.\right. \\
& \left.-\int_{0}^{1} k(\tau) \int_{0}^{\tau}(\tau-s) y(s) \mathrm{d} s \mathrm{~d} \tau\right) \\
& \left.-n \int_{0}^{1} h(\tau) \int_{0}^{\tau}(\tau-s) y(s) \mathrm{d} s \mathrm{~d} \tau\right] .
\end{aligned}
$$

Therefore, (6) has a unique solution

$$
\begin{array}{r}
x(t)=-\int_{0}^{t}(t-s) y(s) \mathrm{d} s+\frac{1}{m \nu+n \mu} \\
\times\left[v t \int_{0}^{1} h(\tau) \int_{0}^{\tau}(\tau-s) y(s) \mathrm{d} s \mathrm{~d} \tau\right. \\
+\mu t\left(\int_{0}^{1}(1-s) y(s) \mathrm{d} s-\int_{0}^{1} k(\tau)\right. \\
\left.\quad \times \int_{0}^{\tau}(\tau-s) y(s) \mathrm{d} s \mathrm{~d} \tau\right) \\
+m\left(\int_{0}^{1}(1-s) y(s) \mathrm{d} s-\int_{0}^{1} k(\tau)\right. \\
\left.\times \int_{0}^{\tau}(\tau-s) y(s) \mathrm{d} s \mathrm{~d} \tau\right) \\
\left.-n \int_{0}^{1} h(\tau) \int_{0}^{\tau}(\tau-s) y(s) \mathrm{d} s \mathrm{~d} \tau\right] .
\end{array}
$$

The unique solution of (6) be expressed as

$$
\begin{aligned}
x(t)= & \int_{0}^{t} s(1-t) y(s) \mathrm{d} s+\int_{t}^{1} t(1-s) y(s) \mathrm{d} s+\frac{1}{m v+n \mu} \\
& \times\left[\mu t \int_{0}^{1} \tau k(\tau) \mathrm{d} \tau \int_{0}^{1}(1-s) y(s) \mathrm{d} s-\mu t\right. \\
& \times \int_{0}^{1}(1-s) y(s) \mathrm{d} s-v t \\
& \times \int_{0}^{1} \tau h(\tau) \mathrm{d} \tau \int_{0}^{1}(1-s) y(s) \mathrm{d} s \\
& +v t \int_{0}^{1} h(\tau) \int_{0}^{\tau}(\tau-s) y(s) \mathrm{d} s \mathrm{~d} \tau+\mu t
\end{aligned}
$$

$\times \int_{0}^{1}(1-s) y(s) \mathrm{d} s-\mu t$

$\times \int_{0}^{1} k(\tau) \int_{0}^{\tau}(\tau-s) y(s) \mathrm{d} s \mathrm{~d} \tau$

$+\int_{0}^{1} \tau h(\tau) \mathrm{d} \tau \int_{0}^{1}(1-s) y(s) \mathrm{d} s-\int_{0}^{1} \tau h(\tau) \mathrm{d} \tau$

$\times \int_{0}^{1} k(\tau) \int_{0}^{\tau}(\tau-s) y(s) \mathrm{d} s \mathrm{~d} \tau-\int_{0}^{1} h(\tau)$

$\times \int_{0}^{\tau}(\tau-s) y(s) \mathrm{d} s \mathrm{~d} \tau+\int_{0}^{1} \tau k(\tau) \mathrm{d} \tau$

$\times \int_{0}^{1} h(\tau) \int_{0}^{\tau}(\tau-s) y(s) \mathrm{d} s \mathrm{~d} \tau-\int_{0}^{1} \tau k(\tau) \mathrm{d} \tau$

$\times \int_{0}^{1} \tau h(\tau) \mathrm{d} \tau \int_{0}^{1}(1-s) y(s) \mathrm{d} s$

$\left.+\int_{0}^{1} \tau h(\tau) \mathrm{d} \tau \int_{0}^{1} \tau k(\tau) \mathrm{d} \tau \int_{0}^{1}(1-s) y(s) \mathrm{d} s\right]$

$=\int_{0}^{t} s(1-t) y(s) \mathrm{d} s+\int_{t}^{1} t(1-s) y(s) \mathrm{d} s+\frac{1}{m v+n \mu}$

$\times\left[\mu t\left(\int_{0}^{1} k(\tau) \int_{0}^{\tau} s(1-\tau) y(s) \mathrm{d} s \mathrm{~d} \tau\right.\right.$

$\left.+\int_{0}^{1} k(\tau) \int_{\tau}^{1} \tau(1-s) y(s) \mathrm{d} s \mathrm{~d} \tau\right)$

$-v t\left(\int_{0}^{1} h(\tau) \int_{0}^{\tau} s(1-\tau) y(s) \mathrm{d} s \mathrm{~d} \tau\right.$

$\left.+\int_{0}^{1} h(\tau) \int_{\tau}^{1} \tau(1-s) y(s) \mathrm{d} s \mathrm{~d} \tau\right)$

$+\left(\int_{0}^{1} h(\tau) \int_{0}^{\tau} s(1-\tau) y(s) \mathrm{d} s \mathrm{~d} \tau\right.$

$\left.+\int_{0}^{1} h(\tau) \int_{\tau}^{1} \tau(1-s) y(s) \mathrm{d} s \mathrm{~d} \tau\right)$

$-\int_{0}^{1} \tau k(\tau) \mathrm{d} \tau$

$\times\left(\int_{0}^{1} h(\tau) \int_{0}^{\tau} s(1-\tau) y(s) \mathrm{d} s \mathrm{~d} \tau\right.$

$\left.+\int_{0}^{1} h(\tau) \int_{\tau}^{1} \tau(1-s) y(s) \mathrm{d} s \mathrm{~d} \tau\right)$

$+\int_{0}^{1} \tau h(\tau) \mathrm{d} \tau$

$\times\left(\int_{0}^{1} k(\tau) \int_{0}^{\tau} s(1-\tau) y(s) \mathrm{d} s \mathrm{~d} \tau\right.$

$\left.\left.+\int_{0}^{1} k(\tau) \int_{\tau}^{1} \tau(1-s) y(s) \mathrm{d} s \mathrm{~d} \tau\right)\right]$ 


$$
\begin{aligned}
& =\int_{0}^{1} G(t, s) y(s) \mathrm{d} s \\
& +\frac{1}{m v+n \mu} \times\left[\mu t \int_{0}^{1} k(\tau) \int_{0}^{1} G(s, \tau) y(s) \mathrm{d} s \mathrm{~d} \tau\right. \\
& -v t \int_{0}^{1} h(\tau) \int_{0}^{1} G(s, \tau) y(s) \mathrm{d} s \mathrm{~d} \tau \\
& +\int_{0}^{1} h(\tau) \int_{0}^{1} G(s, \tau) y(s) \mathrm{d} s \mathrm{~d} \tau \\
& -\int_{0}^{1} \tau k(\tau) \mathrm{d} \tau \int_{0}^{1} h(\tau) \\
& \times \int_{0}^{1} G(s, \tau) y(s) \mathrm{d} s \mathrm{~d} \tau \\
& +\int_{0}^{1} \tau h(\tau) \mathrm{d} \tau \int_{0}^{1} k(\tau) \\
& \left.\times \int_{0}^{1} G(s, \tau) y(s) \mathrm{d} s \mathrm{~d} \tau\right] \\
& =\int_{0}^{1} G(t, s) y(s) \mathrm{d} s \\
& +\frac{m+\mu t}{m v+n \mu} \int_{0}^{1} y(s) \int_{0}^{1} k(\tau) G(s, \tau) \mathrm{d} \tau \mathrm{d} s \\
& +\frac{n-v t}{m v+n \mu} \int_{0}^{1} y(s) \int_{0}^{1} h(\tau) G(s, \tau) \mathrm{d} \tau \mathrm{d} s .
\end{aligned}
$$

Therefore, the unique solution of (6) is $x(t)=$ $\int_{0}^{1} \widetilde{H}(t, s) y(s) \mathrm{d} s$.

Lemma 3 (in [11]). If $h, k \in L^{1}[0,1]$ are nonnegative, then,

(1) $n>v$,

(2) $G(t, s)>0$, for all $t, s \in(0,1), G(t, s) \geq 0$, for all $t, s \in[0,1]$,

(3) Letting $e(t)=t(1-t)$, for all $t, s \in[0,1]$, one has $e(t) e(s) \leq G(t, s) \leq G(t, t)=e(t) \leq 1 / 4$,

(4) Letting $\delta=(0,1 / 2), J_{\delta}=[\delta, 1-\delta]$, for all $t \in$ $J_{\delta}, s \in[0,1]$, we have $G(t, s) \geq \delta G(s, s)$, where $G(t, s)$ is defined by (9).

Lemma 4. If $h, k \in L^{1}[0,1]$ are nonnegative, and $\mu=1-$ $\int_{0}^{1} h(s) d s>0, v=1-\int_{0}^{1} k(s) d s>0$, for function $\widetilde{H}(t, s)$, we have the following properties:

(1) $\widetilde{H}(t, s)>0$, for all $t, s \in(0,1), \widetilde{H}(t, s) \geq$ 0 , for all $t, s \in[0,1]$,

(2) $\rho e(t) e(s) \leq \widetilde{H}(t, s) \leq \eta e(s)$, for all $t, s \in[0,1]$,

(3) $\widetilde{H}(t, s) \geq \delta \widetilde{H}(s, s), t \in J_{\delta}, s \in[0,1]$,

where $\rho=(1 /(m v+n \mu))\left(\mu \int_{0}^{1} e(\tau) k(\tau) d \tau+v \int_{0}^{1} e(\tau) h(\tau) d \tau\right)$, $\eta=(m+n+\mu(1-v)) /(m v+n \mu)$.
Proof.

(1) From (8) and Lemma 3, we get (1).

(2) For all $t, s \in[0,1]$, by (8) and Lemma 3, we have

$$
\begin{aligned}
& \widetilde{H}(t, s)=G(t, s)+\frac{m+\mu t}{m v+n \mu} \int_{0}^{1} k(\tau) G(s, \tau) \mathrm{d} \tau \\
& +\frac{n-v t}{m \nu+n \mu} \int_{0}^{1} h(\tau) G(s, \tau) \mathrm{d} \tau \\
& \geq \frac{m+\mu t}{m v+n \mu} \int_{0}^{1} k(\tau) G(s, \tau) \mathrm{d} \tau \\
& +\frac{n-v t}{m v+n \mu} \int_{0}^{1} h(\tau) G(s, \tau) \mathrm{d} \tau \\
& \geq \frac{e(s)}{m v+n \mu}\left(\mu t \int_{0}^{1} e(\tau) k(\tau) \mathrm{d} \tau\right. \\
& \left.+(1-t) v \int_{0}^{1} e(\tau) h(\tau) \mathrm{d} \tau\right) \\
& \geq \frac{e(s) e(t)}{m \nu+n \mu}\left(\mu \int_{0}^{1} e(\tau) k(\tau) \mathrm{d} \tau+v \int_{0}^{1} e(\tau) h(\tau) \mathrm{d} \tau\right) \\
& =\rho e(t) e(s), \\
& \widetilde{H}(t, s)=G(t, s)+\frac{m+\mu t}{m v+n \mu} \int_{0}^{1} k(\tau) G(s, \tau) \mathrm{d} \tau \\
& +\frac{n-v t}{m \nu+n \mu} \int_{0}^{1} h(\tau) G(s, \tau) \mathrm{d} \tau
\end{aligned}
$$$$
\leq G(s, s)+\frac{m+\mu t}{m v+n \mu} \int_{0}^{1} k(\tau) G(s, s) \mathrm{d} \tau
$$$$
+\frac{n-v t}{m v+n \mu} \int_{0}^{1} h(\tau) G(s, s) \mathrm{d} \tau
$$$$
=e(s)\left(1+\frac{m+\mu t}{m v+n \mu} \int_{0}^{1} k(\tau) \mathrm{d} \tau\right.
$$$$
\left.+\frac{n-v t}{m v+n \mu} \int_{0}^{1} h(\tau) \mathrm{d} \tau\right)
$$$$
\leq \frac{e(s)}{m v+n \mu}(m v+n \mu+(m+\mu)
$$$$
\left.\times \int_{0}^{1} k(\tau) \mathrm{d} \tau+n \int_{0}^{1} h(\tau) \mathrm{d} \tau\right)
$$

$=\eta e(s)$.

(3) For all $t \in J_{\delta}, s \in[0,1]$, by (8) and Lemma 3, we have

$$
\begin{aligned}
\widetilde{H}(t, s)= & G(t, s)+\frac{m+\mu t}{m v+n \mu} \int_{0}^{1} k(\tau) G(s, \tau) \mathrm{d} \tau \\
& +\frac{n-v t}{m v+n \mu} \int_{0}^{1} h(\tau) G(s, \tau) \mathrm{d} \tau
\end{aligned}
$$




$$
\begin{aligned}
\geq & \delta G(s, s)+\frac{\delta(m+\mu s)}{m v+n \mu} \int_{0}^{1} k(\tau) G(s, \tau) \mathrm{d} \tau \\
& +\frac{\delta(n-v s)}{m v+n \mu} \int_{0}^{1} h(\tau) G(s, \tau) \mathrm{d} \tau \\
= & \delta \widetilde{H}(s, s) .
\end{aligned}
$$

Lemma 5. Assume that (A1)-(A3) holds. If $x(t) \in C^{2}[0,1]$ is a solution of the following integral equation

$$
x(t)=(T x)(t) \triangleq \int_{0}^{1} H(t, s) w(s) f\left(s, x(s), x^{\prime \prime}(s)\right) d s,
$$

then $x(t) \in C^{2}[0,1] \cap C^{4}(0,1)$ is a solution of $B V P(1)$, where

$$
\begin{gathered}
H(t, s)=\int_{0}^{1} H_{1}(t, \tau) H_{2}(\tau, s) d \tau \\
H_{1}(t, \tau)=G(t, \tau)+\frac{m_{1}+\mu_{1} t}{m_{1} v_{1}+n_{1} \mu_{1}} \int_{0}^{1} k_{1}(v) G(\tau, v) d v \\
+\frac{n_{1}-v_{1} t}{m_{1} v_{1}+n_{1} \mu_{1}} \int_{0}^{1} h_{1}(v) G(\tau, v) d v \\
H_{2}(\tau, s)= \\
\quad G(\tau, s)+\frac{m_{2}+\mu_{2} \tau}{m_{2} v_{2}+n_{2} \mu_{2}} \int_{0}^{1} k_{2}(v) G(s, v) d v \\
\quad+\frac{n_{2}-v_{2} \tau}{m_{2} v_{2}+n_{2} \mu_{2}} \int_{0}^{1} h_{2}(v) G(s, v) d v \\
m_{1}=\int_{0}^{1} s h_{1}(s) d s, \quad n_{1}=1-\int_{0}^{1} s k_{1}(s) d s \\
m_{2}=\int_{0}^{1} s h_{2}(s) d s, \quad n_{2}=1-\int_{0}^{1} s k_{2}(s) d s .
\end{gathered}
$$

Proof. By using Lemma 2, the conclusion is obvious.

Lemma 6. If (A3) holds, then one has the following three properties:

(1) $H(t, s)>0$, for all $t, s \in(0,1)$,

(2) $0 \leq H(t, s) \leq \eta_{1} \eta_{2} / 16, t, s \in[0,1]$,

(3) $H(1 / 2, s) \geq\left(\rho_{1} \rho_{2} / 120\right) e(s)$, for all $s \in[0,1]$,

where

$$
\begin{aligned}
& \eta_{1}=\frac{m_{1}+n_{1}+\mu_{1}\left(1-v_{1}\right)}{m_{1} v_{1}+n_{1} \mu_{1}}, \\
& \eta_{2}=\frac{m_{2}+n_{2}+\mu_{2}\left(1-v_{2}\right)}{m_{2} v_{2}+n_{2} \mu_{2}},
\end{aligned}
$$

$$
\begin{aligned}
\rho_{1}= & \frac{1}{m_{1} v_{1}+n_{1} \mu_{1}} \\
& \times\left(\mu_{1} \int_{0}^{1} e(\tau) k_{1}(\tau) d \tau+v_{1} \int_{0}^{1} e(\tau) h_{1}(\tau) d \tau\right), \\
\rho_{2}= & \frac{1}{m_{2} v_{2}+n_{2} \mu_{2}} \\
& \times\left(\mu_{2} \int_{0}^{1} e(\tau) k_{2}(\tau) d \tau+v_{2} \int_{0}^{1} e(\tau) h_{2}(\tau) d \tau\right) .
\end{aligned}
$$

Proof. By using Lemma 3, the conclusions for (1) and the first part of (2) are obvious.

By using by (21) and Lemma 3, we have

$$
\begin{aligned}
H_{1}(t, \tau)= & G(t, \tau)+\frac{m_{1}+\mu_{1} t}{m_{1} v_{1}+n_{1} \mu_{1}} \int_{0}^{1} k_{1}(v) G(\tau \\
& +\frac{n_{1}-v_{1} t}{m_{1} v_{1}+n_{1} \mu_{1}} \int_{0}^{1} h_{1}(v) G(\tau, v) \mathrm{d} v \\
\leq & \frac{1}{4}+\frac{m_{1}+\mu_{1} t}{m_{1} v_{1}+n_{1} \mu_{1}} \int_{0}^{1} \frac{1}{4} k_{1}(s) \mathrm{d} s \\
& +\frac{n_{1}-v_{1} t}{m_{1} v_{1}+n_{1} \mu_{1}} \int_{0}^{1} \frac{1}{4} h_{1}(s) \mathrm{d} s \\
\leq & \frac{\eta_{1}}{4} .
\end{aligned}
$$

Similarly, by (22), we get

$$
H_{2}(\tau, s) \leq \frac{\eta_{2}}{4} .
$$

So, by (20), (25), and (26), we concluded

$$
H(t, s)=\int_{0}^{1} H_{1}(t, \tau) H_{2}(\tau, s) \mathrm{d} \tau \leq \frac{\eta_{1} \eta_{2}}{16} .
$$

By using Lemmas 3 and 4 , we have

$$
\begin{aligned}
H\left(\frac{1}{2}, s\right) & =\int_{0}^{1} H_{1}\left(\frac{1}{2}, \tau\right) H_{2}(\tau, s) \mathrm{d} \tau \\
& \geq \frac{\rho_{1}}{4} \int_{0}^{1} e(\tau) H_{2}(\tau, s) \mathrm{d} \tau \\
& \geq \frac{\rho_{1} \rho_{2}}{4} e(s) \int_{0}^{1} e^{2}(\tau) \mathrm{d} \tau=\frac{\rho_{1} \rho_{2}}{120} e(s) .
\end{aligned}
$$

Let $E=C^{2}[0,1]$. Then $E$ is a Banach space with the norm $\|x\|_{2}=\|x\|+\left\|x^{\prime \prime}\right\|$, where $\|x\|=\max _{t \in[0,1]}|x(t)|,\left\|x^{\prime \prime}\right\|=$ $\max _{t \in[0,1]}\left|x^{\prime \prime}(t)\right|$.

For a fixed $\delta=(0,1 / 2), J_{\delta}=[\delta, 1-\delta]$, define the cone $K \subset E$ by

$$
\begin{gathered}
K \triangleq\left\{x \in E: x \geq 0, x^{\prime \prime} \leq 0, \min _{t \in J_{\delta}} x(t) \geq \delta^{2}\|x\|,\right. \\
\left.\max _{t \in J_{\delta}} x^{\prime \prime}(t) \leq-\delta^{2}\left\|x^{\prime \prime}\right\|\right\} .
\end{gathered}
$$


It's easy to prove that $K$ is a closed convex cone, and

$$
|x(t)|+\left|x^{\prime \prime}(t)\right| \geq \delta^{2}\|x\|_{2}, \quad t \in J_{\delta}, \quad x \in K
$$

Lemma 7. If (A1)-(A3) hold, then $T(K) \subset K$, and $T: K \rightarrow$ $K$ is completely continuous.

Proof. By the properties of Green's function, if $x \in C^{2}[0,1]$ then $T x \in C^{2}[0,1]$ and

$$
(T x)^{\prime \prime}(t)=-\int_{0}^{1} H_{2}(t, s) w(s) f\left(s, x(s), x^{\prime \prime}(s)\right) \mathrm{d} s .
$$

For all $x \in K$, we have by (19) and (31)

$$
\begin{gathered}
(T x)(t) \geq 0, \quad(T x)^{\prime \prime}(t) \leq 0 \\
\|(T x)\| \leq \int_{0}^{1} \int_{0}^{1}\left[G(\tau, \tau)+\frac{m_{1}+\mu_{1}}{m_{1} v_{1}+n_{1} \mu_{1}}\right. \\
\times \int_{0}^{1} k_{1}(v) G(v, v) \mathrm{d} v \\
\left.+\frac{n_{1}}{m_{1} v_{1}+n_{1} \mu_{1}} \int_{0}^{1} h_{1}(v) G(v, v) \mathrm{d} v\right] \\
\times H_{2}(\tau, s) \omega(s) f\left(s, x(s), x^{\prime \prime}(s)\right) \mathrm{d} \tau \mathrm{d} s, \\
\left\|(T x)^{\prime \prime}\right\| \leq \int_{0}^{1}\left[G(s, s)+\frac{m_{2}+\mu_{2}}{m_{2} v_{2}+n_{2} \mu_{2}}\right. \\
\times \int_{0}^{1} k_{2}(\tau) G(\tau, \tau) \mathrm{d} \tau \\
\left.\quad+\frac{n_{2}}{m_{2} v_{2}+n_{2} \mu_{2}} \int_{0}^{1} h_{2}(\tau) G(\tau, \tau) \mathrm{d} \tau\right] \\
\times \omega(s) f\left(s, x(s), x^{\prime \prime}(s)\right) \mathrm{d} s .
\end{gathered}
$$

On the other hand, by (19), (33), and Lemma 3, we obtain

$$
\begin{aligned}
& \min _{t \in J_{\delta}}(T x)(t) \\
&=\min _{t \in J_{\delta}} \int_{0}^{1} \int_{0}^{1}\left[G(t, \tau)+\frac{m_{1}+\mu_{1} t}{m_{1} v_{1}+n_{1} \mu_{1}}\right. \\
& \quad \times \int_{0}^{1} k_{1}(v) G(\tau, v) \mathrm{d} v+\frac{n_{1}-v_{1} t}{m_{1} v_{1}+n_{1} \mu_{1}} \\
&\left.\times \int_{0}^{1} h_{1}(v) G(\tau, v) \mathrm{d} v\right] \\
& \quad \times H_{2}(\tau, s) \omega(s) f\left(s, x(s), x^{\prime \prime}(s)\right) \mathrm{d} \tau \mathrm{d} s
\end{aligned}
$$

$$
\begin{aligned}
& \geq \delta \int_{0}^{1} \int_{0}^{1}\left[G(\tau, \tau)+\frac{m_{1}+\mu_{1} \delta}{m_{1} v_{1}+n_{1} \mu_{1}}\right. \\
& \times \int_{0}^{1} k_{1}(v) G(v, v) \mathrm{d} v+\frac{n_{1}-v_{1}(1-\delta)}{m_{1} v_{1}+n_{1} \mu_{1}} \\
& \left.\times \int_{0}^{1} h_{1}(v) G(v, v) \mathrm{d} v\right] \\
& \times H_{2}(\tau, s) \omega(s) f\left(s, x(s), x^{\prime \prime}(s)\right) \mathrm{d} \tau \mathrm{d} s \\
& \geq \delta^{2} \int_{0}^{1} \int_{0}^{1}\left[G(\tau, \tau)+\frac{m_{1}+\mu_{1}}{m_{1} v_{1}+n_{1} \mu_{1}}\right. \\
& \times \int_{0}^{1} k_{1}(v) G(v, v) \mathrm{d} v \\
& \left.+\frac{n_{1}}{m_{1} v_{1}+n_{1} \mu_{1}} \int_{0}^{1} h_{1}(v) G(v, v) \mathrm{d} v\right] \\
& \times H_{2}(\tau, s) \omega(s) f\left(s, x(s), x^{\prime \prime}(s)\right) \mathrm{d} \tau \mathrm{d} s \\
& \geq \delta^{2}\|T x\| \text {. }
\end{aligned}
$$

Similarly, by (31), (34), and Lemma 3, we obtain

$$
\begin{aligned}
\max _{t \in J_{\delta}}(T x)^{\prime \prime}(t) & \\
= & -\min _{t \in J_{\delta}} \int_{0}^{1} H_{2}(t, s) w(s) f\left(s, x(s), x^{\prime \prime}(s)\right) \mathrm{d} s \\
= & -\min _{t \in J_{\delta}} \int_{0}^{1}\left[G(t, s)+\frac{m_{2}+\mu_{2} t}{m_{2} v_{2}+n_{2} \mu_{2}}\right. \\
& \times \int_{0}^{1} k_{2}(\tau) G(s, \tau) \mathrm{d} \tau \\
& \left.+\frac{n_{2}-v_{2} t}{m_{2} v_{2}+n_{2} \mu_{2}} \int_{0}^{1} h_{2}(\tau) G(s, \tau) \mathrm{d} \tau\right] \\
\leq & -\delta^{2} \int_{0}^{1}[s) f\left(s, x(s), x^{\prime \prime}(s)\right) \mathrm{d} s \\
\leq & -\delta^{2}\left\|(T x)^{\prime \prime}\right\| \cdot \frac{m_{2}+\mu_{2}}{m_{2} v_{2}+n_{2} \mu_{2}} \\
\times & \times \int_{0}^{1} k_{2}(\tau) G(\tau, \tau) \mathrm{d} \tau \\
\left.+\frac{n_{2}}{m_{2} v_{2}+n_{2} \mu_{2}} \int_{0}^{1} h_{2}(\tau) G(\tau, \tau) \mathrm{d} \tau\right] & f\left(s, x(s), x^{\prime \prime}(s)\right) \mathrm{d} s
\end{aligned}
$$

So $T x \subset K$, and then $T(K) \subset K$.

Clearly, the operator $T$ is continuous; thus by the ArzelaAscoli theorem, it follows that $T$ is completely continuous. 


\section{Main Results}

For convenience, we introduce the following notations:

$$
\begin{gathered}
K_{r}=\left\{x \in K:\|x\|_{2} \leq r\right\}, \partial K_{r}=\left\{x \in K:\|x\|_{2}=r\right\}, \\
\bar{K}_{r, R}=\left\{x \in K: r \leq\|x\|_{2} \leq R\right\}, \\
f^{\beta}=\limsup _{|x|+|y| \rightarrow \beta} \max _{t \in[0,1]} \frac{f(t, x, y)}{|x|+|y|}, \\
f_{\beta}=\liminf _{|x|+|y| \rightarrow \beta} \min _{t \in[0,1]} \frac{f(t, x, y)}{|x|+|y|}, \\
M_{\beta}=\max _{t \in[0,1], 0<|x|+|y| \leq \beta} f(t, x, y), \\
L=\left(\frac{\eta_{1} \eta_{2}}{16}+\frac{\eta_{2}}{4}\right) \int_{0}^{1} w(s) \mathrm{d} s, \\
N=\left(\frac{\rho_{1} \rho_{2}}{120}+\frac{\rho_{2}}{4}\right) \delta^{2} \int_{\delta}^{1-\delta} e(s) w(s) \mathrm{d} s .
\end{gathered}
$$

Theorem 8. Assume that (A1)-(A3) hold. In addition, one supposes that one of the following conditions is satisfied

$\left(H_{1}\right) L f(t, x, y)<|x|+|y|$, for all $|x|+|y| \neq 0, t \in[0,1]$,

$\left(H_{2}\right) N f(t, x, y)>|x|+|y|$, for all $|x|+|y| \neq 0, t \in[0,1]$.

Then, the BVP (1) has no positive solution.

Proof. Case 1. The condition $\left(H_{1}\right)$ holds. Assume towards a contraction that $x^{*}(t)$ is a positive solution of BVP (1), from the proof of Lemma 7 and (29), then $x^{*} \in K, x^{*}(t)>0$ for $0<t<1$. By Lemma 6 and (29), we have

$$
\begin{aligned}
\left\|x^{*}\right\|= & \max _{t \in[0,1]}\left|x^{*}(t)\right|=\max _{t \in[0,1]}\left|T x^{*}(t)\right| \\
= & \max _{t \in[0,1]}\left|\int_{0}^{1} H(t, s) w(s) f\left(s, x^{*}(s), x^{*^{\prime \prime}}(s)\right) \mathrm{d} s\right| \\
& <\frac{\eta_{1} \eta_{2}}{16 L} \int_{0}^{1} w(s)\left(\left|x^{*}(s)\right|+\left|x^{*^{\prime \prime}}(s)\right|\right) \mathrm{d} s \\
\leq & \frac{\eta_{1} \eta_{2}}{16 L}\left\|x^{*}\right\|_{2} \int_{0}^{1} w(s) \mathrm{d} s, \\
\left\|x^{*^{\prime \prime}}\right\|= & \max _{t \in[0,1]}\left\|x^{*^{\prime \prime}}(t)\right\|=\max _{t \in[0,1]}\left|\left(T x^{*}\right)^{\prime \prime}(t)\right| \\
= & \max _{t \in[0,1]}\left|\int_{0}^{1} H_{2}(t, s) w(s) f\left(s, x^{*}(s), x^{*^{\prime \prime}}(s)\right) \mathrm{d} s\right| \\
& <\frac{\eta_{2}}{4 L} \int_{0}^{1} w(s)\left(\left|x^{*}(s)\right|+\left|x^{*^{\prime \prime}}(s)\right|\right) \mathrm{d} s \\
\leq & \frac{\eta_{2}}{4 L}\left\|x^{*}\right\|_{2} \int_{0}^{1} w(s) \mathrm{d} s .
\end{aligned}
$$

So, $\left\|x^{*}\right\|_{2}=\left\|x^{*}\right\|+\left\|x^{* \prime \prime}\right\|<(1 / L)\left(\eta_{1} \eta_{2} / 16+\eta_{2} / 4\right)\left\|x^{*}\right\|_{2}$ $\int_{0}^{1} w(s) \mathrm{d} s=\left\|x^{*}\right\|_{2}$, which is a contradiction.
Case 2. The condition $\left(\mathrm{H}_{2}\right)$ holds. Assume towards a contraction that $x^{* *}(t)$ is a positive solution of BVP (1), from the proof of Lemma 7 and (29), then $x^{* *} \in K, x^{* *}(t)>0$, for $0<t<1$. By Lemmas 4 and 6 and (30), we have

$$
\begin{aligned}
\left\|x^{* *}\right\|= & \max _{t \in[0,1]}\left|x^{* *}(t)\right|=\max _{t \in[0,1]}\left|T x^{* *}(t)\right| \\
\geq & \int_{0}^{1} H\left(\frac{1}{2}, s\right) w(s) f\left(s, x^{* *}(s), x^{* *^{\prime \prime}}(s)\right) \mathrm{d} s \\
& >\frac{\rho_{1} \rho_{2}}{120 N} \int_{0}^{1} e(s) w(s)\left(\left|x^{* *}(s)\right|+\left|x^{* *^{\prime \prime}}(s)\right|\right) \mathrm{d} s \\
\geq & \frac{\rho_{1} \rho_{2}}{120 N} \int_{\delta}^{1-\delta} e(s) w(s)\left(\left|x^{* *}(s)\right|+\left|x^{* *^{\prime \prime}}(s)\right|\right) \mathrm{d} s \\
\geq & \frac{\rho_{1} \rho_{2}}{120 N} \delta^{2}\left\|x^{* *}\right\|_{2} \int_{\delta}^{1-\delta} e(s) w(s) \mathrm{d}, \\
\left\|x^{* *}\right\| & =\max _{t \in[0,1]}\left|x^{* *^{\prime \prime}}(t)\right|=\max _{t \in[0,1]}\left|\left(T x^{* *}\right)^{\prime \prime}(t)\right| \\
= & \max _{t \in[0,1]}\left|\int_{0}^{1} H_{2}(t, s) w(s) f\left(s, x^{* *}(s), x^{* *^{\prime \prime}}(s)\right) \mathrm{d} s\right| \\
\geq & \int_{0}^{1} H_{2}\left(\frac{1}{2}, s\right) w(s) f\left(s, x^{* *}(s), x^{* *^{\prime \prime}}(s)\right) \mathrm{d} s \\
> & \frac{\rho_{2}}{4 N} \int_{0}^{1} e(s) w(s)\left(\left|x^{* *}(s)\right|+\left|x^{* *^{\prime \prime}}(s)\right|\right) \mathrm{d} s \\
&
\end{aligned}
$$

So, $\left\|x^{* *}\right\|_{2}=\left\|x^{* *}\right\|+\left\|x^{* * \| \prime}\right\|>(1 / N)\left(\rho_{1} \rho_{2} / 120+\rho_{2} / 4\right) \delta^{2}$ $\left\|x^{* *}\right\|_{2} \int_{\delta}^{1-\delta} e(s) w(s) \mathrm{d} s=\left\|x^{* *}\right\|_{2}$, which is a contradiction, and this completes the proof.

Theorem 9. Assume that (A1)-(A3) hold. In addition, one supposes that one of the following conditions is satisfied

$$
\begin{aligned}
& \left(H_{3}\right) L f^{0}<1<N f_{\infty}, \\
& \left(H_{4}\right) L f^{\infty}<1<N f_{0} .
\end{aligned}
$$

Then, the BVP (1) has at least one positive solution.

Proof. Case 1 . The condition $\left(\mathrm{H}_{3}\right)$ holds.

Considering $L f^{0}<1$, then there exists $r_{1}>0$, such that $f(t, x, y) \leq\left(f^{0}+\varepsilon_{1}\right)(|x|+|y|)$, for $t \in[0,1],(x, y) \in\{(x, y):$ $\left.0<|x|+|y| \leq r_{1}\right\}$, where $\varepsilon_{1}>0$, satisfies $L\left(f^{0}+\varepsilon_{1}\right) \leq 1$.

Then, for $t \in[0,1], x \in \partial K_{r_{1}}$, by (19), (26), and Lemma 6, we have

$$
\begin{aligned}
\|T x\|_{2} & =\|T x\|+\left\|(T x)^{\prime \prime}\right\| \\
& \leq\left(\frac{\eta_{1} \eta_{2}}{16}+\frac{\eta_{2}}{4}\right) \int_{0}^{1} w(s) f\left(s, x(s), s^{\prime \prime}(s)\right) \mathrm{d} s
\end{aligned}
$$




$$
\begin{aligned}
\leq & \left(\frac{\eta_{1} \eta_{2}}{16}+\frac{\eta_{2}}{4}\right)\left(f^{0}+\varepsilon_{1}\right) \\
& \times \int_{0}^{1} w(s)\left(|x(s)|+\left|x^{\prime \prime}(s)\right|\right) \mathrm{d} s \\
\leq & \left(\frac{\eta_{1} \eta_{2}}{16}+\frac{\eta_{2}}{4}\right)\left(f^{0}+\varepsilon_{1}\right)\|x\|_{2} \int_{0}^{1} w(s) \mathrm{d} s \leq\|x\|_{2},
\end{aligned}
$$

which means that

$$
\|T x\|_{2} \leq\|x\|_{2}, \quad \text { for } x \in \partial K_{r_{1}} .
$$

Next, turning to $N f_{\infty}>1$, then there exists $\bar{R}_{1}>0$, such that $f(t, x, y) \geq\left(f_{\infty}-\varepsilon_{2}\right)(|x|+|y|)$, for $t \in[0,1],(x, y) \in$ $\left\{(x, y): 0<|x|+|y|>\bar{R}_{1}\right\}$, where $\varepsilon_{2}>0$, satisfies $N\left(f_{\infty}-\right.$ $\left.\varepsilon_{2}\right) \geq 1$.

Let $R_{1}=\max \left\{2 r_{1}, \bar{R}_{1} / \delta^{2}\right\}$, for all $t \in J_{\delta}, x \in \partial K_{R_{1}}$. By (30) and Lemma 6, we have

$$
\begin{aligned}
\|T x\|_{2}= & \|T x\|+\left\|(T x)^{\prime \prime}\right\| \geq\left|T x\left(\frac{1}{2}\right)\right|+\left|(T x)^{\prime \prime}\left(\frac{1}{2}\right)\right| \\
\geq & \left(\frac{\rho_{1} \rho_{2}}{120}+\frac{\rho_{2}}{4}\right) \int_{0}^{1} e(s) w(s) f\left(s, x(s), s^{\prime \prime}(s)\right) \mathrm{d} s \\
\geq & \left(\frac{\rho_{1} \rho_{2}}{120}+\frac{\rho_{2}}{4}\right)\left(f_{\infty}-\varepsilon_{2}\right) \\
& \times \int_{0}^{1} e(s) w(s)\left(|x(s)|+\left|x^{\prime \prime}(s)\right|\right) \mathrm{d} s \\
\geq & \left(\frac{\rho_{1} \rho_{2}}{120}+\frac{\rho_{2}}{4}\right)\left(f_{\infty}-\varepsilon_{2}\right) \delta^{2}\|x\|_{2} \\
& \times \int_{\delta}^{1-\delta} e(s) w(s) \mathrm{d} s \geq\|x\|_{2}
\end{aligned}
$$

which means that

$$
\|T x\|_{2} \geq\|x\|_{2}, \quad \text { for } x \in \partial K_{R_{1}} .
$$

Case 2. The condition $\left(\mathrm{H}_{4}\right)$ holds.

Considering $N f^{0}>1$, then there exists $r_{2}>0$, such that $f(t, x, y) \geq\left(f^{0}-\varepsilon_{3}\right)(|x|+|y|)$, for $t \in[0,1],(x, y) \in\{(x, y)$ : $\left.0<|x|+|y| \leq r_{2}\right\}$, where $\varepsilon_{3}>0$, satisfies $N\left(f^{0}-\varepsilon_{3}\right) \geq 1$.

Then, for $t \in J_{\delta}, x \in \partial K_{r_{2}}$, by (30) and Lemma 6, we have

$$
\begin{aligned}
\|T x\|_{2} \geq & \left|T x\left(\frac{1}{2}\right)\right|+\left|(T x)^{\prime \prime}\left(\frac{1}{2}\right)\right| \\
\geq & \left(\frac{\rho_{1} \rho_{2}}{120}+\frac{\rho_{2}}{4}\right)\left(f_{0}-\varepsilon_{3}\right) \\
& \times \int_{0}^{1} e(s) w(s)\left(|x(s)|+\left|x^{\prime \prime}(s)\right|\right) \mathrm{d} s \\
\geq & \left(\frac{\rho_{1} \rho_{2}}{120}+\frac{\rho_{2}}{4}\right)\left(f_{0}-\varepsilon_{3}\right) \delta^{2}\|x\|_{2} \\
& \times \int_{\delta}^{1-\delta} e(s) w(s) \mathrm{d} s \geq\|x\|_{2},
\end{aligned}
$$

which means that

$$
\|T x\|_{2} \geq\|x\|_{2}, \quad \text { for } x \in \partial K_{r_{2}} .
$$

Next, turning to $L f^{\infty}<1$, then there exists $\bar{R}_{2}>0$, such that $f(t, x, y) \leq\left(f^{\infty}+\varepsilon_{4}\right)(|x|+|y|)$, for $t \in[0,1],(x, y) \in$ $\left\{(x, y):|x|+|y|>\bar{R}_{2}\right\}$, where $\varepsilon_{4}>0$, satisfies $L\left(f^{\infty}+\varepsilon_{4}\right) \leq 1$.

Let

$$
M_{\bar{R}_{2}}=\max _{t \in[0,1], 0<|x|+|y| \leq \bar{R}_{2}} f(t, x, y)
$$

for $t \in[0,1], f(t, x, y) \leq M_{\bar{R}_{2}}+\left(f^{\infty}+\varepsilon_{4}\right)(|x|+|y|)$.

Let $R_{2}=\max \left\{2 r_{2}, \bar{R}_{2}, L M_{\bar{R}_{2}} /\left(1-L\left(f^{\infty}+\varepsilon_{4}\right)\right)\right\}$, for $t \in$ $[0,1], x \in \partial K_{R_{2}}$. By (26) and Lemma 6, we have

$$
\begin{aligned}
\|T x\|_{2} \leq & \left(\frac{\eta_{1} \eta_{2}}{16}+\frac{\eta_{2}}{4}\right) \int_{0}^{1} w(s) f\left(s, x(s), s^{\prime \prime}(s)\right) \mathrm{d} s \\
\leq & \left(\frac{\eta_{1} \eta_{2}}{16}+\frac{\eta_{2}}{4}\right) \\
& \times\left(M_{\bar{R}_{2}} \int_{0}^{1} w(s) \mathrm{d} s+\left(f^{\infty}+\varepsilon_{4}\right)\right. \\
& \left.\times \int_{0}^{1} w(s)\left(|x(s)|+\left|x^{\prime \prime}(s)\right|\right) \mathrm{d} s\right) \\
\leq & L M_{\bar{R}_{2}}+\|x\|_{2} E\left(f^{\infty}+\varepsilon_{4}\right) \leq R_{2}-L\left(f^{\infty}+\varepsilon_{4}\right) R_{2} \\
& +\|x\|_{2} L\left(f^{\infty}+\varepsilon_{4}\right)=\|x\|_{2},
\end{aligned}
$$

which means that

$$
\|T x\|_{2} \leq\|x\|_{2}, \quad \text { for } x \in \partial K_{R_{2}} .
$$

Applying Lemma 1 to (41) and (43), or (45), and (48) yields that $T$ has a fixed point $x_{*} \in \bar{K}_{r_{i}, R_{i}}(i=1,2)$ with $x_{*}(t) \geq \delta\left\|x_{*}\right\|_{2}, t \in(0,1)$. Thus, it follows that BVP (1) has at least one positive solution, and the theorem is proved.

Theorem 10. Assume that (A1)-(A3) hold, as do the following two conditions:

$\left(H_{5}\right) N f_{0}>1$ and $N f_{\infty}>1$,

$\left(H_{6}\right)$ there exists $b>0$, such that $\max _{t \in[0,1], 0<|x|+|y| \leq b} f(t$, $x, y)<b / L$.

Then, the BVP (1) has at least two positive solutions $x_{*}(t), x_{* *}(t)$, which satisfy

$$
0<\left\|x_{*}\right\|_{2}<b<\left\|x_{* *}\right\|_{2} .
$$

Proof. We choose $r, R$ with $0<r<b<R$.

If $N f_{0}>1$, then by the proof of (45), we have

$$
\|T x\|_{2} \geq\|x\|_{2}, \quad \text { for }\|x\|_{2}=r .
$$

If $N f_{\infty}>1$, then by the proof of (43), we have

$$
\|T x\|_{2} \geq\|x\|_{2}, \quad \text { for }\|x\|_{2}=R \text {. }
$$


On the other hand, by $\left(H_{6}\right)$, for $x \in \partial K_{b}$, we have

$$
\begin{aligned}
\|T x\|_{2} & \leq\left(\frac{\eta_{1} \eta_{2}}{16}+\frac{\eta_{2}}{4}\right) \int_{0}^{1} w(s) f\left(s, x(s), s^{\prime \prime}(s)\right) \mathrm{d} s \\
& \leq\left(\frac{\eta_{1} \eta_{2}}{16}+\frac{\eta_{2}}{4}\right) M_{b} \int_{0}^{1} w(s) \mathrm{d} s=L M_{b},
\end{aligned}
$$

where $M_{b}=\max _{t \in[0,1], 0<|x|+|y| \leq b} f(t, x, y)<b / L$.

By (52), we have

$$
\|T x\|_{2}<b=\|x\|_{2} .
$$

Applying Lemma 1 to (50), (51), and (53) yields that $T$ has a fixed point $x_{*} \in \bar{K}_{r, b}$, and a fixed point $x_{* *} \in \bar{K}_{b, R}$. Thus it follows that BVP (1) has at least two positive solutions $x_{*}$ and $x_{* *}$. Noticing (53), we have $\left\|x_{*}\right\|_{2} \neq b$ and $\left\|x_{* *}\right\|_{2} \neq b$. Therefore, (49) holds, and the proof is complete.

Theorem 11. Assume that (A1)-(A3) hold, as do the following two conditions:

$\left(H_{7}\right) L f^{0}<1$ and $L f^{\infty}<1$,

$\left(H_{8}\right)$ there exist $\delta \in(0,1 / 2)$ and $B>0$ such that $f(t, x, y)>$ $\delta^{2} B / N$ for all $t \in J_{\delta}, x \in\left[\delta^{2} B, B\right]$ and $y \in$ $\left[-B,-\delta^{2} B\right]$.

Then, the BVP (1) has at least two positive solutions $x^{*}(t), x^{* *}(t)$, which satisfy

$$
0<\left\|x^{*}\right\|_{2}<B<\left\|x^{* *}\right\|_{2} \text {. }
$$

Proof. We choose $\bar{r}, \bar{R}$ with $0<\bar{r}<B<\bar{R}$.

If $L f_{0}<1$, then by the proof of (41), we have

$$
\|T x\|_{2} \leq\|x\|_{2}, \quad \text { for }\|x\|_{2}=\bar{r} .
$$

If $L f_{\infty}<1$, then by the proof of (48), we have

$$
\|T x\|_{2} \leq\|x\|_{2}, \quad \text { for }\|x\|_{2}=\bar{R} .
$$

On the other hand, by $\left(H_{8}\right)$, for $x \in \partial K_{B}$, we have

$$
\begin{aligned}
\|T x\|_{2} \geq & \left(\frac{\rho_{1} \rho_{2}}{120}+\frac{\rho_{2}}{4}\right) \int_{0}^{1} e(s) w(s) \\
& \times f\left(s, x(s), s^{\prime \prime}(s)\right) \mathrm{d} s>B=\|x\|_{2} .
\end{aligned}
$$

Applying Lemma 1 to (55), (56), and (57) yields that $T$ has a fixed point $x_{*} \in \bar{K}_{\bar{r}, B}$, and a fixed point $x_{* *} \in \bar{K}_{B, \bar{R}}$. Thus it follows that BVP (1) has at least two positive solutions $x_{*}$ and $x_{* *}$. Noticing (57), we have $\left\|x_{*}\right\|_{2} \neq B$ and $\left\|x_{* *}\right\|_{2} \neq B$. Therefore, (54) holds, and the proof is complete.

\section{Example}

Example 12. Consider the following fourth-order BVP

$$
\begin{gathered}
x^{(4)}(t)=\frac{1}{\sqrt{t}} f\left(t, x(t), x^{\prime \prime}(t)\right), \quad t \in(0,1), \\
x(0)=\int_{0}^{1} s x(s) \mathrm{d} s, \quad x(1)=\int_{0}^{1} s x(s) \mathrm{d} s, \\
x^{\prime \prime}(0)=\int_{0}^{1} s^{2} x^{\prime \prime}(s) \mathrm{d} s, \quad x^{\prime \prime}(1)=\int_{0}^{1} s^{2} x^{\prime \prime}(s) \mathrm{d} s .
\end{gathered}
$$

By calculation, we obtain

$$
\begin{gathered}
\mu_{1}=1-\int_{0}^{1} s \mathrm{~d} s=\frac{1}{2}, \quad v_{1}=1-\int_{0}^{1} s \mathrm{~d} s=\frac{1}{2}, \\
m_{1}=\int_{0}^{1} s^{2} \mathrm{~d} s=\frac{1}{3}, \quad n_{1}=1-\int_{0}^{1} s^{2} \mathrm{~d} s=\frac{2}{3}, \\
\mu_{2}=1-\int_{0}^{1} s^{2} \mathrm{~d} s=\frac{2}{3}, \quad v_{2}=1-\int_{0}^{1} s^{2} \mathrm{~d} s=\frac{2}{3}, \\
m_{2}=\int_{0}^{1} s^{3} \mathrm{~d} s=\frac{1}{4}, \quad n_{2}=1-\int_{0}^{1} s^{3} \mathrm{~d} s=\frac{3}{4}, \\
\eta_{1}=\frac{5}{2}, \quad \eta_{2}=\frac{11}{6}, \quad \rho_{1}=\frac{1}{6}, \\
L=\frac{143}{96}, \quad \delta=\frac{1}{3}, \\
N=\frac{181 \sqrt{3}}{2187000}(3 \sqrt{2}-2) \approx 0.00032 .
\end{gathered}
$$

(1) About the nonexistence of positive solution, we consider BVP (58) with

$$
f(t, x, y)=k_{1} e^{t}(|x|+|y|)+k_{2}|\sin (|x|+|y|)|,
$$

where $k_{1}$ and $k_{2}$ are two positive real numbers, then

$$
\frac{f(t, x, y)}{|x|+|y|}<e k_{1}+k_{2}, \quad \frac{f(t, x, y)}{|x|+|y|}>k_{1} \text {. }
$$

If $e k_{1}+k_{2}<1 / L$ or $k_{1}>1 / N$, by Theorem 8 , BVP (58) has no positive solution.

(2) About the existence of positive solution, we consider BVP (58) with

$f(t, x, y)=k_{1} e^{t-1}(|x|+|y|)+k_{2}|\sin (|x|+|y|)|$,

where $k_{1}$ and $k_{2}$ are two positive real numbers, then

$$
\begin{gathered}
f_{0}=\liminf _{|x|+|y| \rightarrow 0} \min _{t \in[0,1]} \frac{f(t, x, y)}{|x|+|y|}=\frac{k_{1}}{e}+k_{2}, \\
f^{\infty}=\limsup _{|x|+|y| \rightarrow \infty} \max _{t \in[0,1]} \frac{f(t, x, y)}{|x|+|y|}=k_{1} .
\end{gathered}
$$

If $k_{1}<1 / L$ and $k_{2}>1 / N$, then we have $L f^{\infty}<1<$ $N f_{0}$. By Theorem 9, BVP (58) has at least one positive solution.

(3) About the multiplicity of positive solution, we consider BVP (58) with

$$
\begin{aligned}
& f(t, x, y) \\
& \quad= \begin{cases}e^{t}\left|\sin k_{1}(|x|+|y|)\right|, & |x|+|y| \leq b, \\
e^{t}\left|\sin k_{1}(|x|+|y|)\right| & (1+t)(|x|+|y|-b)^{2} \\
+k_{2} \frac{(1+|x|+|y|+1}{\mid}, & |x|+|y|>b,\end{cases}
\end{aligned}
$$


where $k_{1}$ and $k_{2}$ are two positive real numbers, then

$$
\begin{aligned}
f_{0} & =\liminf _{|x|+|y| \rightarrow 0} \min _{t \in[0,1]} \frac{f(t, x, y)}{|x|+|y|}=k_{1}, \\
f_{\infty} & =\limsup _{|x|+|y| \rightarrow \infty} \max _{t \in[0,1]} \frac{f(t, x, y)}{|x|+|y|}=2 k_{2} .
\end{aligned}
$$

If $k_{1}>1 / N$ and $k_{2}>1 / 2 N$, then we have $N f_{0}>1$ and $N f_{\infty}>1$.

Furthermore, letting $b>L M_{b}>143 e / 96$, then we have $M_{b}<b / L$. By Theorem 10, BVP (58) has at least two positive solutions.

Remark 13. In [4, 7-10], the existence of solutions for fourthorder ordinary differential equations BVP has been treated but did not discuss problems with singularities. Although, [11] seems to have considered the existence of positive solutions for the fourth-order BVP with integral boundary conditions for the singularity allowed to appear at $t=0$ and/or $t=1$. However, in [11], only the boundary conditions $x(0)=0, x^{\prime \prime}(0)=0$, or $x(1)=0, x^{\prime \prime}(1)=0$ have been considered. It is clear that the boundary conditions of Example 12 are $x(0) \neq 0, x^{\prime \prime}(0) \neq 0$, and $x(1) \neq 0, x^{\prime \prime}(1) \neq 0$. Hence, we generalize the fourth-order ordinary differential equations BVP.

\section{Acknowledgments}

This work is partially supported by the National Natural Science Foundation of PR China (10971045), the Natural Science Foundation of Hebei Province (A2013208147) and (A2011208012), and the Foundation of Hebei University of Science and Technology (XL201246). The authors thank the referee for his/her careful reading of the paper and useful suggestions that have greatly improved this paper.

\section{References}

[1] X. Hao, L. Liu, and Y. Wu, "Positive solutions for nonlinear $n$ thorder singular nonlocal boundary value problems," Boundary Value Problems, vol. 2007, Article ID 74517, 10 pages, 2007.

[2] X. Hao, L. Liu, Y. Wu, and Q. Sun, "Positive solutions for nonlinear $n$ th-order singular eigenvalue problem with nonlocal conditions," Nonlinear Analysis: Theory, Methods \& Applications, vol. 73, no. 6, pp. 1653-1662, 2010.

[3] J. R. L. Webb and G. Infante, "Positive solutions of nonlocal boundary value problems: a unified approach," Journal of the London Mathematical Society, vol. 74, no. 3, pp. 673-693, 2006.

[4] R. Ma and L. Xu, "Existence of positive solutions of a nonlinear fourth-order boundary value problem," Applied Mathematics Letters, vol. 23, no. 5, pp. 537-543, 2010.

[5] A. Cabada and R. R. Enguiça, "Positive solutions of fourth order problems with clamped beam boundary conditions," Nonlinear Analysis: Theory, Methods \& Applications, vol. 74, no. 10, pp. 3112-3122, 2011.

[6] F. Xu, "Three symmetric positive solutions of fourth-order nonlocal boundary value problems," Electronic Journal of Qualitative Theory of Differential Equations, no. 96, pp. 1-11, 2011.
[7] R. Ma and T. Chen, "Existence of positive solutions of fourthorder problems with integral boundary conditions," Boundary Value Problems, vol. 2011, Article ID 297578, 17 pages, 2011.

[8] Z. Bai, "Positive solutions of some nonlocal fourth-order boundary value problem," Applied Mathematics and Computation, vol. 215, no. 12, pp. 4191-4197, 2010.

[9] J. R. L. Webb, G. Infante, and D. Franco, "Positive solutions of nonlinear fourth-order boundary-value problems with local and non-local boundary conditions," Proceedings of the Royal Society of Edinburgh, vol. 138, no. 2, pp. 427-446, 2008.

[10] X. Zhang, M. Feng, and W. Ge, "Existence results for nonlinear boundary-value problems with integral boundary conditions in Banach spaces," Nonlinear Analysis: Theory, Methods \& Applications, vol. 69, no. 10, pp. 3310-3321, 2008.

[11] X. Zhang and W. Ge, "Positive solutions for a class of boundaryvalue problems with integral boundary conditions," Computers \& Mathematics with Applications, vol. 58, no. 2, pp. 203-215, 2009.

[12] G. Infante and P. Pietramala, "A cantilever equation with nonlinear boundary conditions," Electronic Journal of Qualitative Theory of Differential Equations, no. 15, pp. 1-14, 2009.

[13] P. Pietramala, "A note on a beam equation with nonlinear boundary conditions," Boundary Value Problems, vol. 2011, Article ID 376782, 14 pages, 2011.

[14] J. R. L. Webb and G. Infante, "Non-local boundary value problems of arbitrary order," Journal of the London Mathematical Society, vol. 79, no. 1, pp. 238-258, 2009.

[15] J. R. L. Webb and G. Infante, "Semi-positone nonlocal boundary value problems of arbitrary order," Communications on Pure and Applied Analysis, vol. 9, no. 2, pp. 563-581, 2010.

[16] M. A. Krasnoselśkiǔ, Positive Solutions of Operator Equations, P. Noordhoff, Groningen, The Netherlands, 1964. 


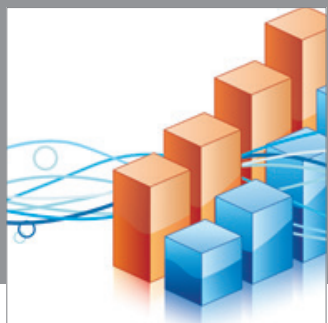

Advances in

Operations Research

mansans

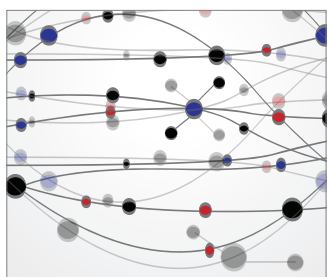

The Scientific World Journal
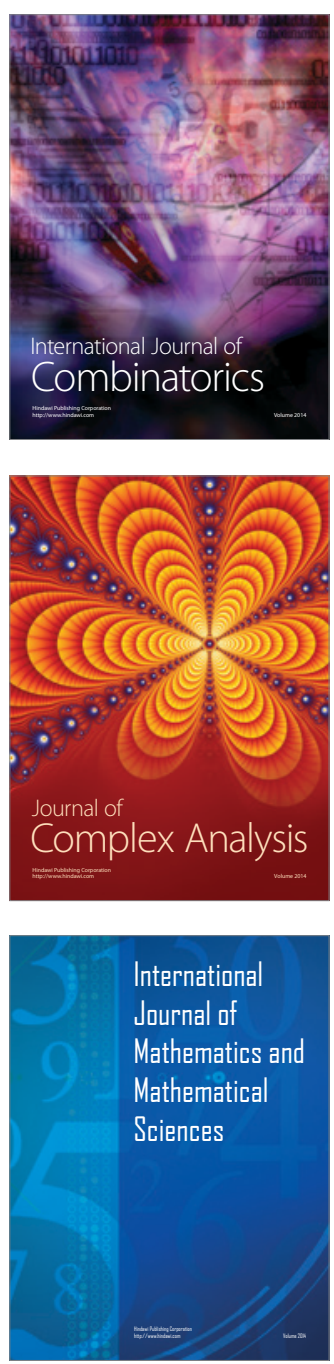
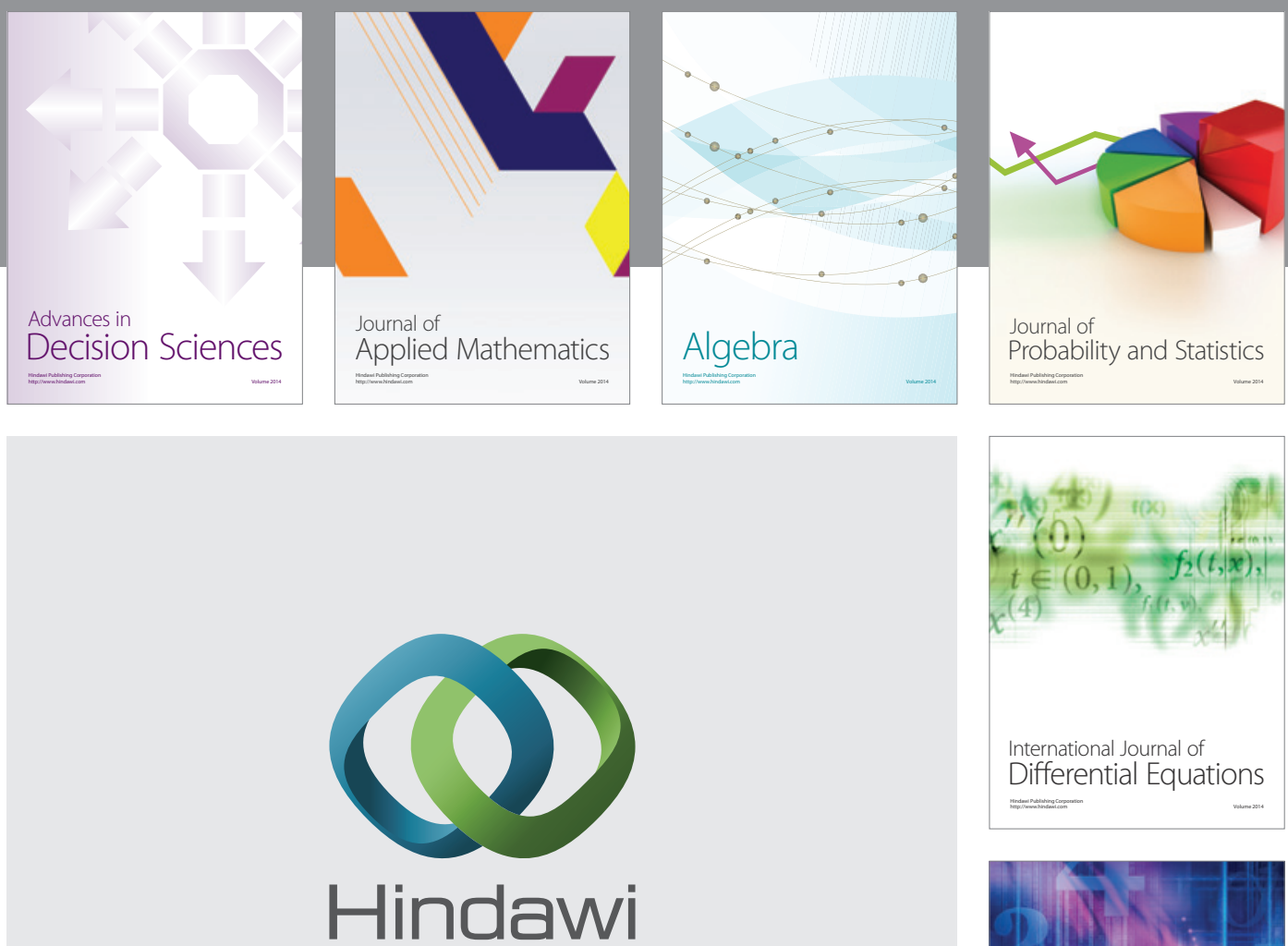

Submit your manuscripts at http://www.hindawi.com
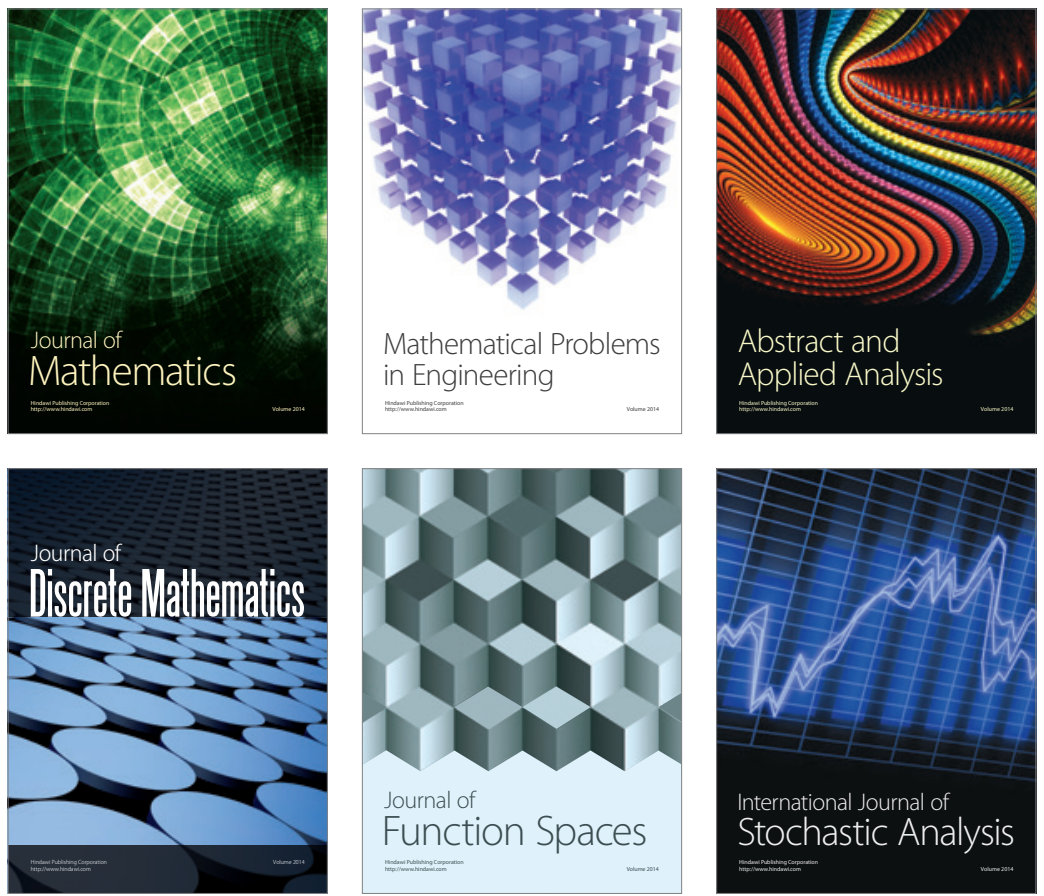

Journal of

Function Spaces

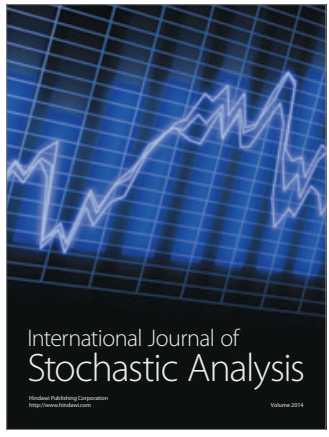

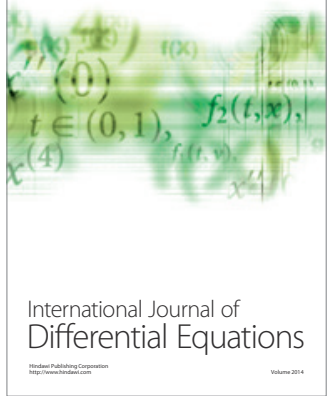
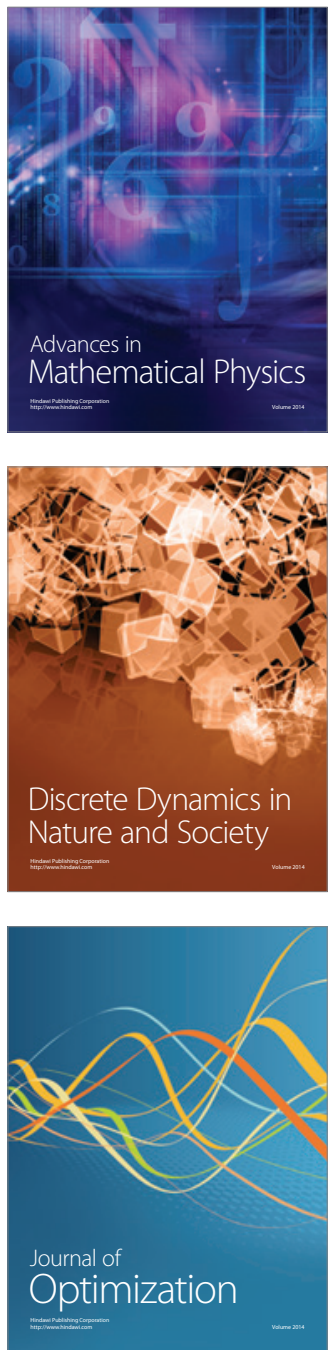